\title{
On some Multidimensional Fractional Integral Operators Involving Multivariable I-Function
}

\author{
Yashwant Singh, Nanda Kulkarni* \\ Department of Mathematics, \\ Government College, Kaladera, Jaipur, \\ Rajasthan, India \\ Department of Mathematics, \\ Maharani Lakshmi Ammanni College for Women \\ Bangalore, Karnataka, India
}

Keywords: Fractional Integral Operators, Multivariable I-function, Multidimensional Integral Transforms

Abstract. In the present paper, we study certain multidimensional fractional integral operators involving a general $I$-function in their kernel. We give five basic properties of these operators, and then establish two theorems and two corollaries, which are believed to be new. These basic theorems exhibit structural relationships between the multidimensional integral transforms. The one- and two-dimensional analogues of these results, which are new and of interest in themselves, can easily be deduced. Special cases of these latter theorems will give rise to certain known results obtained from time to time by several earlier authors.

\section{Introduction}

Fractional integral operators have been defined and studied by various authors notably by Riemann and Liouville [4], Weyl [4], Erdelyi [1,2], Kober [7], Sneddon [15], Kalla [10], Saxena [13], Srivastava and Buschmann [16] etc. These operators play an important role in the theory of integral equations and problems concerning Mathematical Physics. In this paper, we shell study the following multidimensional fractional integral operators having the general function $N$ as their kernel. Also, for the sake of brevity, we shall use the symbol $f(x)$ to represent $f\left(x_{1}, x_{2}, ., x_{\mathrm{r}}\right)$.

The multivariable $I$-function introduced by Prasad [12] will be define and represent it in the following manner:

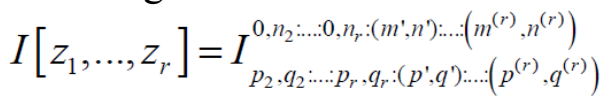

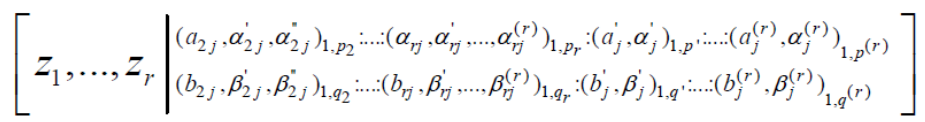

$$
\begin{aligned}
& =\frac{1}{(2 \pi w)^{r}} \int_{L_{1}} \ldots \int_{L_{r}} \phi_{1}\left(s_{1}\right) \ldots \phi_{r}\left(s_{r}\right) \psi\left(s_{1}, \ldots, s_{r}\right) z_{1}^{s_{1}}, \ldots, z_{r}^{s_{r}} d s_{1} \ldots d s_{r}
\end{aligned}
$$

Where

$$
\phi_{i}\left(s_{i}\right)=\frac{\prod_{j=1}^{m^{(i)}} \Gamma\left(b_{j}^{(i)}-\beta_{j}^{(i)} s_{i}\right) \prod_{j=1}^{n^{(i)}} \Gamma\left(1-a_{j}^{(i)}+\alpha_{j}^{(i)} s_{i}\right)}{\prod_{j=m^{(i)}+1}^{q^{(i)}} \Gamma\left(1-b_{j}^{(i)}+\beta_{j}^{(i)} s_{i}\right) \prod_{j=n^{(i)}+1}^{p^{(i)}} \Gamma\left(a_{j}^{(i)}-\alpha_{j}^{(i)} s_{i}\right)} \forall i \in(1,2, \ldots, r)
$$




$$
\begin{gathered}
\psi\left(s_{1}, \ldots, s_{r}\right)=\frac{\prod_{j=1}^{n_{2}} \Gamma\left(1-a_{2 j}+\sum_{i=1}^{2} \alpha_{2 j}^{(i)} s_{i}\right) \prod_{j=1}^{n_{3}} \Gamma\left(1-a_{3 j}+\sum_{i=1}^{3} \alpha_{3 j}^{(i)} s_{i}\right)}{\prod_{j=n_{2}+1}^{p_{2}} \Gamma\left(a_{2 j}-\sum_{i=1}^{2} \alpha_{2 j}^{(i)} s_{i}\right) \prod_{j=n_{3}+1}^{p_{3}} \Gamma\left(a_{3 j}-\sum_{i=1}^{3} \alpha_{3 j}^{(i)} s_{i}\right)} \\
\ldots \prod_{j=1}^{n_{r}} \Gamma\left(1-a_{r j}+\sum_{i=1}^{r} \alpha_{r j}^{(i)} s_{i}\right) \\
\ldots \prod_{j=n_{r}+1}^{p_{r}} \Gamma\left(a_{r j}-\sum_{i=1}^{r} \alpha_{r j}^{(i)} s_{i}\right) \prod_{j=1}^{q_{2}} \Gamma\left(1-b_{2 j}-\sum_{i=1}^{2} \beta_{2 j}^{(i)} s_{i}\right) \ldots \prod_{j=1}^{q_{r}} \Gamma\left(1-b_{r j}-\sum_{i=1}^{r} \beta_{r j}^{(i)} s_{i}\right)
\end{gathered}
$$

$\alpha_{j}^{(i)}, \beta_{j}^{(i)}, \alpha_{k j}^{(i)}, \beta_{k j}^{(i)}(i=1, \ldots, r)(k=1, \ldots, r)$ are positive numbers, $a_{j}^{(i)}, b_{j}^{(i)}(i=1, \ldots, r), a_{k j}, b_{k j}(k=2, \ldots, r)$ are complex numbers and here $m^{(i)}, n^{(i)}, p^{(i)}, q^{(i)}(i=1, \ldots, r), n_{k}, p_{k}, q_{k}(k=2, \ldots, r)$ are non-negative integers where $0 \leq m^{(i)} \leq q^{(i)}, 0 \leq n^{(i)} \leq p^{(i)}, q^{k} \geq 0,0 \leq n_{k} \leq p_{k}$. Here $(i)$ denotes the numbers of dashes. The contours $L_{i}$ in the complex $s_{i}$-plane is of the Mellin-Barnes type which runs from $-w \infty$ to $+w \infty$ with indentations, if necessary, to ensure that all the poles of $\Gamma\left(b_{j}^{(i)}-\beta_{j}^{(i)} s_{i}\right)\left(j=1, \ldots, m^{(i)}\right)$ are separated from those of $\Gamma\left(1-a_{r j}+\sum_{i=1}^{r} \alpha_{r j}^{(i)} s_{i}\right)\left(j=1, \ldots, n_{r}\right)$.

For further details and asymptotic expansion of the $I$-function one can refer by Prasad [12].

In what follows, the multivariable $I$-function defined by [12] will be represented in the contracted notation:

$$
I_{p_{2}, q_{2}: \ldots . p_{r}, q_{r}:\left(p^{\prime}, q^{\prime}\right): \ldots .:\left(p^{(r)}, q^{(r)}\right)}^{0, n_{2}: \ldots: 0, n_{r}:\left(m^{\prime}, n^{\prime}\right) \cdots:\left(m^{(r)}, n^{(r)}\right)}\left[z_{1}, \ldots, Z_{r}\right]
$$

Or simply by $I\left[z_{1}, \ldots, z_{r}\right]$

We introduce the fractional integration operators by means of the following integral equations:

$$
\begin{aligned}
& Y\{f(x)\}=Y\left\{f(x) ; t_{1}, \ldots, t_{r} ; \gamma_{1}, \ldots, \gamma_{r}\right\}=\prod_{i=1}^{r}\left(t_{i}^{\gamma_{i}-1}\right) \\
& \int_{0}^{t_{1}} \ldots \int_{0}^{t_{r}} \prod_{i=1}^{r}\left\{\left(x_{i}\right)^{\gamma_{i}} \phi\left(\frac{x_{1}}{t_{1}}, \ldots, \frac{x_{r}}{t_{r}}\right)\right\} f(x) d x_{1} \ldots d x_{r}
\end{aligned}
$$

And

$$
\begin{aligned}
& N\{f(x)\}=N\left\{f(x) ; t_{1}, \ldots, t_{r} ; \delta_{1}, \ldots, \delta_{r}\right\}=\prod_{i=1}^{r}\left(t_{i}^{\delta_{i}}\right) \\
& \int_{t_{1}}^{\infty} \ldots \int_{t_{r}}^{\infty} \prod_{i=1}^{r}\left\{\left(x_{i}\right)^{-\delta_{i}-1} \phi\left(\frac{t_{1}}{x_{1}}, \ldots, \frac{t_{r}}{x_{r}}\right)\right\} f(x) d x_{1} \ldots d x_{r}
\end{aligned}
$$

Where the kernel $\phi$ is such that the above integrals make sense. The above operators exist under the following conditions:

(i) $p_{i} \leq 1, q_{i}<\infty, \frac{1}{p_{i}}+\frac{1}{q_{i}}=1, i=1,2, \ldots, r$

(ii) $\operatorname{Re}\left(\gamma_{i}\right)>-\frac{1}{q_{i}} ; \operatorname{Re}\left(\delta_{i}\right)>-\frac{1}{p_{i}} ; i=1,2, \ldots, r$

(iii) $f(x) \in L_{p_{i}}((0, \infty), \ldots,(0, \infty)) ; i=1,2, \ldots, r$

(iv) $0 \leq m^{(i)} \leq q^{(i)}, 0 \leq n^{(i)} \leq p^{(i)}, q^{k} \geq 0,0 \leq n_{k} \leq p_{k}$

The following special case of the multidimensional fractional integral operators involving product of Gauss's hypergeometric functions ([14],p.153,eq. (i) and (ii))will be used in Section 3.

$I\{f(x)\}=\prod_{i=1}^{r}\left\{\frac{t_{i}^{\gamma_{i}-1}}{\Gamma\left(1-a_{i}\right)}\right\} \int_{0}^{t_{1}} \ldots \int_{0}^{t_{r}} \prod_{i=1}^{r}\left\{\left(x_{i}\right)_{2}^{\gamma_{i}} F_{1}\left(\alpha_{i}, \beta_{i}+m ; \beta_{i} ; \frac{x_{i}}{t_{i}}\right)\right\} f(x) d x_{1} \ldots d x_{r}$ 
And

$K\{f(x)\}=\prod_{i=1}^{r}\left\{\frac{t_{i}^{\delta_{i}}}{\Gamma\left(1-\alpha_{i}\right)}\right\} \int_{t_{1}}^{\infty} \ldots \int_{t_{r}}^{\infty} \prod_{i=1}^{r}\left\{\left(x_{i}\right)^{-\delta_{i}-1}{ }_{2} F_{1}\left(\alpha_{i}, \beta_{i}+m ; \beta_{i} ; \frac{t_{i}}{x_{i}}\right)\right\} f(x) d x_{1} \ldots d x_{r}$

The conditions of existence of these operators follow easily from the conditions given in the paper referred to above.

The generalized multidimensional integral transform $T$, defined below, will also be required during the course of our study:

$T\left\{f(x) ; s_{1}, \ldots, s_{r}\right\}=\int_{0}^{\infty} \ldots \int_{0}^{\infty} k\left(s_{1} x_{1}, \ldots, s_{r} x_{r}\right) f(x) d x_{1} \ldots d x_{r}$

Where $k\left(s_{1}, x_{1}, \ldots, s_{r}, x_{r}\right)$ is the kernel of the transform $T$ and the multiple integral occurring in the equation (1.8) is assumed to be convergent.

The following multivariable $I$-function transform will also be used in the sequel:

$I\left\{f(x) ; s_{1}, \ldots, S_{r}\right\}=\int_{0}^{\infty} \ldots \int_{0}^{\infty} I_{p_{2}, q_{2}: \ldots .: p_{r}, q_{r}:\left(p^{\prime}, q^{\prime}\right) \ldots . .\left(p^{(r)}, q^{(r)}\right)}^{0, n_{2} \ldots: 0, n_{r}:\left(m^{\prime}, n^{\prime}\right) \cdots \cdot\left(m^{(r)},{ }^{(r)}\right)}$

$\left[\begin{array}{c|c}s_{1} x_{1} & \left(a_{2 j}, \alpha_{2 j}^{\prime}, \alpha_{2 j}^{\prime}\right)_{1, p_{2}} \ldots .\left(\alpha_{r j}, \alpha_{r j}^{\prime}, \ldots, \alpha_{r j}^{(r)}\right)_{1, p_{r}}:\left(a_{j}^{\prime}, \alpha_{j}^{\prime}\right)_{1, p} \cdots \ldots\left(a_{j}^{(r)}, \alpha_{j}^{(r)}\right)_{1, p}^{(r)} \\ \vdots & \left(b_{2 j}, \beta_{2 j}^{\prime}, \beta_{2 j}^{\prime}\right)_{1, q_{2}} \ldots . .\left(b_{r j}, \beta_{r j}^{\prime}, \ldots, \beta_{r j}^{(r)}\right)_{1, q_{r}}:\left(b_{j}^{\prime}, \beta_{j}^{\prime}\right)_{1, q^{\prime}} \ldots .\left(b_{j}^{(r)}, \beta_{j}^{(r)}\right)_{1, q^{(r)}}^{(r)}\end{array}\right] f(x) d x_{1} \ldots d x_{r}$

The transform defined above will be denoted symbolically as follows:

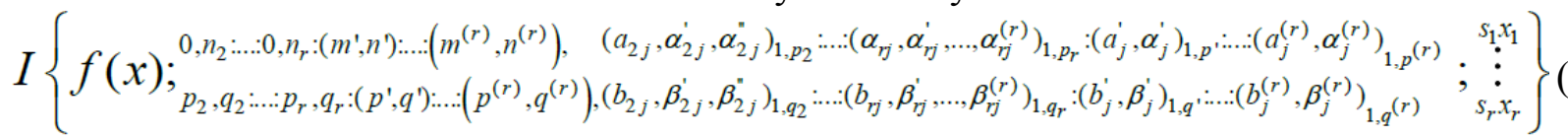

\section{Some Basic Properties}

Property 1. If $f(x) \in L_{p_{i}}((0, \infty), \ldots,(0, \infty)) ; 1 \leq p_{i} \leq 2$ (or $f(x) \in M_{p_{i}}((0, \infty), \ldots,(0, \infty)) ; p_{i}>2$, where $M_{p i}((0, \infty), \ldots,(0, \infty))$ denotes the class of all functions $f(x) \in L_{p_{i}}((0, \infty), \ldots,(0, \infty)) ; p_{i}>2$, which are the inverse Mellin transforms of functions belonging to $L_{q_{i}}((-\infty, \infty), \ldots,(-\infty, \infty)) ; \operatorname{Re}\left(\gamma_{i}\right)>\frac{1}{q_{i}}, \operatorname{Re}\left(\delta_{i}\right)>\frac{1}{p_{i}}, \frac{1}{p}+\frac{1}{q}=1(i=1,2, \ldots, r) \quad$ and $\quad$ the multidimensional Mellin transform of the function $f(x)$ exists, then

(a) $M\left[Y\left\{f(x) ; t_{1}, \ldots, t_{r} ; \gamma_{1}, \ldots, \gamma_{r}\right\} ; s_{1}, \ldots, s_{r}\right]=$

$$
M\left[f(x) ; s_{1}, \ldots, s_{r}\right] N\left\{1 ; t_{1}, \ldots, t_{r} ; \gamma_{1}-s_{1}+1, \ldots, \gamma_{r}-s_{r}+1\right\}
$$

(b) $M\left[N\left\{f(x) ; t_{1}, \ldots, t_{r} ; \delta_{1}, \ldots, \delta_{r}\right\} ; s_{1}, \ldots, s_{r}\right]=$

$$
M\left[f(x) ; s_{1}, \ldots, s_{r}\right] Y\left\{1 ; t_{1}, \ldots, t_{r} ; \delta_{1}+s_{1}+1, \ldots, \delta_{r}+s_{r}+1\right\}
$$

Where the symbol $M$ occurring in (2.1) and (2.2) stands for the multidimensional Mellin transform defined in the following way:

$M\left\{f(x) ; s_{1}, \ldots, s_{r}\right\}=\int_{0}^{\infty} \ldots \int_{0}^{\infty} f(x) \prod_{i=1}^{r}\left(x_{i}^{s_{i}-1}\right) d x_{1} \ldots d x_{r}$

Provided that the multiple integral involved in (2.3) exists.

Proof: To prove (2.1), we use (2.3)and (1.4) to obtain

$M[Y\{f(x)\}]=\int_{0}^{\infty} \ldots \int_{0}^{\infty}\left(t_{i}^{s_{i}-1}\right)\left\{\int_{0}^{t_{1}} \ldots \int_{0}^{t_{r}} \prod_{i=1}^{r}\left(x_{i}^{\gamma_{i}}\right) f(x) \phi\left(\frac{x_{1}}{t_{1}}, \ldots, \frac{x_{r}}{t_{r}}\right)\right\} d t_{1} \ldots d t_{r}$

Now interchanging the orders of $t_{i}$ and $x_{i}(i=1,2, \ldots, r)$ integrals in (2.4), which is easily seen to be permissible under the conditions stated with (2.1) and interpreting the results thus obtained with the help of (1.5), we arrive at the required result (2.1).

The result (2.2) can be established in a similar manner. 
Property 2. If $f(x) \in L_{p_{i}}((0, \infty), \ldots,(0, \infty)) ; 1 \leq p_{i} \leq 2, \quad g(x) \in L_{q_{i}}((0, \infty), \ldots,(0, \infty)) ;$ $\operatorname{Re}\left(\gamma_{i}\right)>\max \left(-\frac{1}{p_{i}},-\frac{1}{q_{i}}\right),(i=1,2, \ldots, r)$, then

$\int_{0}^{\infty} \ldots \int_{0}^{\infty} f(x) Y\{g(x)\} d x_{1} \ldots d x_{r}=\int_{0}^{\infty} \ldots \int_{0}^{\infty} g(x) N\{f(x)\} d x_{1} \ldots d x_{r}$

Provided that the multiple integrals involved in (2.5) are absolutely convergent.

Property 3.

(a) $Y\left\{f(w x) ; t_{1}, \ldots, t_{r} ; \gamma_{1}, \ldots, \gamma_{r}\right\}=Y\left\{f(x) ; t_{1} w_{1}, \ldots, t_{r} w_{r} ; \gamma_{1}, \ldots, \gamma_{r}\right\}$

(b) $N\left\{f(w x) ; t_{1}, \ldots, t_{r} ; \delta_{1}, \ldots, \delta_{r}\right\}=W\left\{f(x) ; t_{1} w_{1}, \ldots, t_{r} w_{r} ; \delta_{1}, \ldots, \delta_{r}\right\}$

Property 4.

(a) $Y\left\{f(1 / x) ; t_{1}, \ldots, t_{r} ; \gamma_{1}, \ldots, \gamma_{r}\right\}=Y\left\{f(x) ; 1 / t_{1}, \ldots, 1 / t_{r} ; \gamma_{1}+1, \ldots, \gamma_{r}+1\right\}$

(b) $N\left\{f(1 / x) ; t_{1}, \ldots, t_{r} ; \delta_{1}, \ldots, \delta_{r}\right\}=W\left\{f(x) ; 1 / t_{1}, \ldots, 1 / t_{r} ; \delta_{1}-1, \ldots, \delta_{r}-1\right\}$

Property 5.

(a) $Y\left\{\prod_{i=1}^{r}\left(x_{i}^{\beta_{i}}\right) f(x) ; t_{1}, \ldots, t_{r} ; \gamma_{1}, \ldots, \gamma_{r}\right\}=$

$\prod_{i=1}^{r}\left(t_{i}^{\beta_{i}}\right) Y\left\{f(x) ; t_{1}, \ldots, t_{r} ; \gamma_{1}+\beta_{1}, . ., \gamma_{r}+\beta_{r}\right\}$

(b) $N\left\{\prod_{i=1}^{r}\left(x_{i}^{\beta_{i}}\right) f(x) ; t_{1}, \ldots, t_{r} ; \delta_{1}, \ldots, \delta_{r}\right\}=$

$\prod_{i=1}^{r}\left(t_{i}^{\beta_{i}}\right) N\left\{f(x) ; t_{1}, \ldots, t_{r} ; \delta_{1}-\beta_{1}, . ., \delta_{r}-\beta_{r}\right\}$

Provided that the multiple integrals involved in (2.10) and (2.11) are absolutely convergent.

The proofs of the properties 2 to 5 are straight forward and follow from the definitions (1.4) and (1.5) of the multidimensional fractional integral operators involved therein

\section{Relationship Between Multidimensional Fractional Integral operators and Multidimensional Integral Transforms}

In this section, we shall establish two most general theorems exhibiting interconnections between the fractional integral operators $Y$ and $N$ defined by (1.4) and (1.5) respectively and the integral transform $T$ defined by (1.8). Next, we give two interesting corollaries interconnecting the multidimensional fractional integral operators defined by (1.6) and (1.7) and the multidimensional I-function transform defined by (1.9).

Theorem 1. If

$\tau\left(s_{1}, . ., s_{r}\right)=T\left\{\psi\left(u^{\rho}\right) g(u) ; s_{1}, \ldots, s_{r}\right\}=$

$\int_{0}^{\infty} \ldots \int_{0}^{\infty} k(s u) \psi\left(u^{\rho}\right) g(u) d u_{1} \ldots d u_{r}$

And

$\psi\left(t_{1}, \ldots, t_{r}\right)=Y\left\{f\left(x^{\sigma}\right) ; t_{1}, \ldots, t_{r}\right\}=$

$\left.\prod_{i=1}^{r}\left(x_{i}^{-\gamma_{i}-1}\right) \int_{0}^{t_{1}} \ldots \int_{0}^{t_{r}} f\left(x^{\sigma}\right) \prod_{i=1}^{r}\left(x_{i}^{\gamma_{i}}\right) \phi\left(\frac{x_{1}}{t_{1}}, \ldots, \frac{x_{r}}{t_{r}}\right)\right\} d x_{1} \ldots d x_{r}$

Then

$$
\begin{aligned}
& \tau\left(s_{1}, \ldots, s_{r}\right)=\frac{1}{\rho_{1} \ldots \rho_{r}} \int_{0}^{\infty} \ldots \int_{0}^{\infty} f\left(x^{\sigma}\right) \\
& \theta\left(s_{1}, \ldots, s_{r}, x_{1}, \ldots, x_{r}, \rho_{1}, \ldots, \rho_{r}\right) d x_{1} \ldots d x_{r}
\end{aligned}
$$




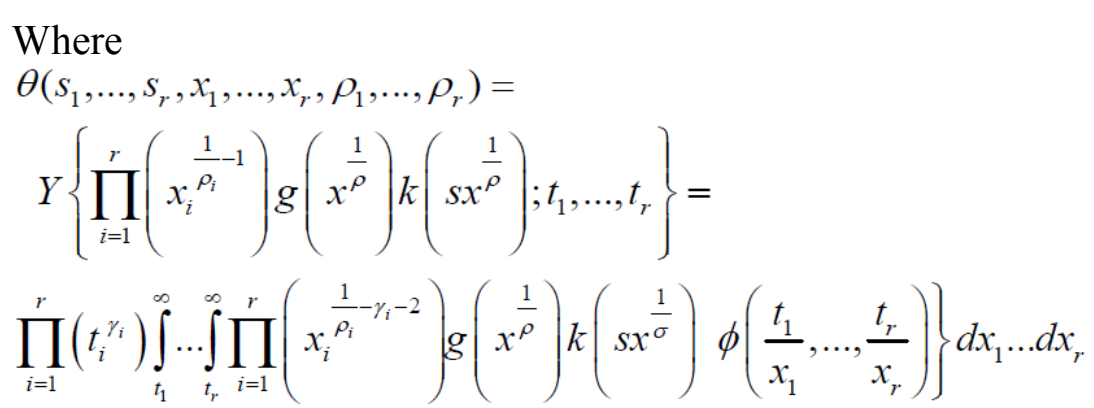

Where $\rho_{i}$ and $\sigma_{i}(i=1,2, \ldots, r)$ are non zero real numbers of the same sign and all the integrals involved in equations (3.1) to (3.4) are assumed to be absolutely convergent. Also in (3.4) $k\left(s x^{\frac{1}{\sigma}}\right)$ stands for $k\left(s_{1} x_{1}^{\frac{1}{\rho_{1}}}, \ldots, s_{r} x_{r}^{\frac{1}{\rho_{r}}}\right)$ and so on.

Proof: Applying the formula (2.5) to the pair of equations (3.2) and (3.4), we get

$\int_{0}^{\infty} \ldots \int_{0}^{\infty} f\left(x^{\sigma}\right) \theta\left(s_{1}, \ldots, s_{r}, x_{1}, \ldots, x_{r}, \rho_{1}, \ldots, \rho_{r}\right) d x_{1} \ldots d x_{r}=$
$\int_{0}^{\infty} \ldots \int_{0}^{\infty} \prod_{i=1}^{r}\left(x_{i}^{\frac{1}{\rho_{i}}-1}\right) g\left(x^{\frac{1}{\rho}}\right) k\left(s x^{\frac{1}{\sigma}}\right) \psi(x) d x_{1} \ldots d x_{r}$

Now changing the variables of integrations on the right hand side of (3.5) slightly and interpreting the result thus obtained with the help of (3.1), we easily arrive at (3.3) after a little simplification.

If in the above theorem, we replace $\rho_{i}$ and $h_{i}(i=1,2, \ldots, r)$ and take

$g(x)=\prod_{i=1}^{r}\left\{x_{i}^{h_{i}\left(c_{i}+1\right)-1}\right\}$,

$\phi(x)=\prod_{i=1}^{r}\left\{\frac{1}{\Gamma\left(1-\alpha_{i}\right)}{ }_{2} F_{1}\left(\alpha_{i}, \beta_{i}+m ; \beta_{i} ; x_{i}\right)\right\}$

And $T$ to be multidimensional $I$-function transform defined by (1.8), the right hand side of equation (3.4) assumes the following form:

$$
\begin{aligned}
& \prod_{i=1}^{r}\left\{\frac{t_{i}^{\gamma_{i}}}{\Gamma\left(1-\alpha_{i}\right)}\right\} \int_{t_{1}}^{\infty} \ldots \int_{t_{r}}^{\infty} \prod_{i=1}^{r}\left\{x_{i}^{c_{i}-\gamma_{i}-1}{ }_{2} F_{1}\left(\alpha_{i}, \beta_{i}+m ; \beta ; \frac{t_{i}}{x_{i}}\right)\right\} \\
& I\left(s_{1} x_{1}^{\frac{1}{h_{1}}}, \ldots, s_{r} x_{r}^{\frac{1}{h_{r}}}\right) d x_{1} \ldots d x_{r}
\end{aligned}
$$

On evaluation the above integral with the help of known results ([4], p.398, eq.(2)) and ([3], p.105, eq.(1)) and the definition of multivariable I-function (1.1) and substituting the values of $\theta\left(s_{1}, \ldots, s_{r}, x_{1}, \ldots, x_{r}, \rho_{1}, \ldots, \rho_{r}\right)$ thus obtained, in the right hand side of equation (3.3), we obtain the following interesting corollary with the help of equations (3.1) and (3.3).

Corollary 1. If $h_{i}>0, \operatorname{Re}\left(1-\alpha_{i}\right)>m, m \in W$, (the set of whole number) $\sigma_{i}(i=1,2, \ldots, r)$ are non-zero real numbers of the same sign.

$\beta_{i} \neq 0,-1,-2, \ldots, \operatorname{Re}\left(C_{i}\right) \geq 0$

$f(x)=\stackrel{o\left(x_{i}^{A_{i}}\right), \text { forsmallvaluesof } x_{i}}{o\left(x_{i}^{B_{i}} e^{-C_{i} x_{i}}\right) \text { forl argevalues of } x_{i}} ; i=1,2, \ldots, r$

The multivariable $I$-function satisfy the conditions corresponding appropriately to those given by Srivastava and Panda ([18],p.130, eqs. (1.6) and (1.10)), then 
$I\left\{\prod_{i=1}^{r} x_{i}^{h_{i}\left(c_{i}+1\right)-1} \psi\left(x^{h}\right) ;{ }_{p_{2}, q_{2}: \ldots .: p_{r}, q_{r}:\left(p^{\prime}, q^{\prime}\right) \ldots . .\left(p^{(r)}, q^{(r)}\right),}^{0, n_{2}: \ldots .: n_{n}:\left(m^{\prime}, n^{\prime}\right): \ldots:\left(m^{(r)}, n^{(r)}\right),}\right.$

$\left(a_{2 j}, \alpha_{2 j}^{\prime}, \alpha_{2 j}^{\prime \prime}\right)_{1, p_{2}}: \ldots . .\left(\alpha_{r j}, \alpha_{r j}^{\prime}, \ldots, \alpha_{r j}^{(r)}\right)_{1, p_{r}}:\left(a_{j}^{\prime}, \alpha_{j}^{\prime}\right)_{1, p^{\prime}-1} \ldots . .\left(a_{j}^{(r)}, \alpha_{j}^{(r)}\right)_{1, p}(r){ }_{-1}$

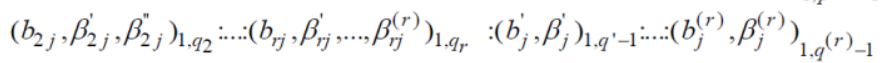

$$
\left.\begin{array}{l}
\left(1-\alpha_{1}+\gamma_{1}-c_{1}, \frac{1}{h_{1}}\right), \ldots,\left(1-\alpha_{r}+\gamma_{r}-c_{r}, \frac{1}{h_{r}}\right) \\
\left(1-\beta_{1}+\gamma_{1}-m-c_{1}, \frac{1}{h_{1}}\right), \ldots,\left(1-\beta_{r}+\gamma_{r}-m-c_{r}, \frac{1}{h_{r}}\right) ; S_{1}, \ldots, S_{r}
\end{array}\right\}=
$$

$\frac{1}{\prod_{i=1}^{r}\left(\beta_{i}\right)_{m}} I\left\{\prod_{i=1}^{r} x_{i}^{h_{i}\left(c_{i}+1\right)-1} f\left(x^{h \sigma}\right) ;{ }_{p_{2}, q_{2} \ldots . p_{r}, q_{r}:\left(p^{\prime}, q^{\prime}\right) \ldots . . .\left(p^{(r)}, q^{(r)}\right),}^{0, n_{2}: n_{r}:\left(m^{\prime}, n^{\prime}\right) \ldots .:\left(m^{(r)}, n^{(r)}\right),}\right.$,

$\left(a_{2 j}, \alpha_{2 j}^{\prime}, \alpha_{2 j}^{\prime \prime}\right)_{1, p_{2}}: \ldots .:\left(\alpha_{r j}, \alpha_{r j}^{\prime}, \ldots, \alpha_{r j}^{(r)}\right)_{1, p_{r}}:\left(a_{j}^{\prime}, \alpha_{j}^{\prime}\right)_{1, p^{\prime}-1} \ldots .:\left(a_{j}^{(r)}, \alpha_{j}^{(r)}\right)_{1, p^{(r)}-1}$

$\left(b_{2 j}, \beta_{2 j}^{\prime}, \beta_{2 j}^{\prime \prime}\right)_{1, q_{2}} \ldots . .\left(b_{r j}, \beta_{r j}^{\prime}, \ldots, \beta_{r j}^{(r)}\right)_{1, q_{r}}:\left(b_{j}^{\prime}, \beta_{j}^{\prime}\right)_{1, q^{\prime}-1} \ldots . .\left(b_{j}^{(r)}, \beta_{j}^{(r)}\right)_{1, q^{(r)}}{ }_{-1}$

$$
\left.\begin{array}{l}
\left(\gamma_{1}-c_{1}, \frac{1}{h_{1}}\right), \ldots,\left(\gamma_{r}-c_{r}, \frac{1}{h_{r}}\right) \\
\left(1-\beta_{1}+\gamma_{1}-c_{1}, \frac{1}{h_{1}}\right), \ldots,\left(1-\beta_{r}+\gamma_{r}-c_{r}, \frac{1}{h_{r}}\right) ; s_{1}, \ldots, s_{r}
\end{array}\right\}
$$

Where

$$
\begin{aligned}
& \psi\left(t_{1}, \ldots, t_{r}\right)=I\left\{f(x) ; t_{1}, \ldots, t_{r}\right\}=\prod_{i=1}^{r}\left\{\frac{t_{i}^{\gamma_{i}}}{\Gamma\left(1-\alpha_{i}\right)}\right\} \\
& \int_{0}^{t_{1}} \ldots \int_{0}^{t_{r}} \prod_{i=1}^{r}\left\{x_{i}^{\gamma_{i}} F_{1}\left(\alpha_{i}, \beta_{i}+m ; \beta_{i} ; \frac{x_{i}}{t_{i}}\right)\right\} f\left(x^{\sigma}\right) d x_{1} \ldots d x_{r}
\end{aligned}
$$

Provided that

$\operatorname{Re}\left(c_{i}-\gamma_{i}+\frac{1}{h_{i}} \frac{b_{j}^{(i)}-1}{B_{j}^{(i)}}\right)<0 ; 1 \leq j \leq n^{(i)}$

$\operatorname{Re}\left(\sigma_{i} B_{i}+c_{i}+1+\frac{1}{h_{i}} \frac{b_{j}^{(i)}-1}{B_{j}^{(i)}}\right)<0 ; 1 \leq j \leq n^{(i)}$

$\operatorname{Re}\left(\sigma_{i} A_{i}+c_{i}+1+\frac{1}{h_{i}} \frac{d_{k}^{(i)}-1}{D_{k}^{(i)}}\right)<0 ; 1 \leq k \leq m^{(i)}$

$\operatorname{Re}\left(\sigma_{i} A_{i}+\gamma_{i}+1\right)>0 ; i=1,2, \ldots, r$

Theorem 2. If

$$
\tau\left(s_{1}, . ., s_{r}\right)=T\left\{\psi\left(u^{\rho}\right) g(u) ; s_{1}, \ldots, s_{r}\right\}=
$$

$\int_{0}^{\infty} \ldots \int_{0}^{\infty} k(s u) \psi\left(u^{\rho}\right) g(u) d u_{1} \ldots d u_{r}$

And

$$
\begin{aligned}
\psi\left(t_{1}, \ldots, t_{r}\right) & =N\left\{f\left(x^{\sigma}\right) ; t_{1}, \ldots, t_{r}\right\}= \\
\prod_{i=1}^{r}\left(t_{i}^{\gamma_{i}}\right) & \left.\int_{t_{1}}^{\infty} \ldots \int_{t_{r}}^{\infty} f\left(x^{\sigma}\right) \prod_{i=1}^{r}\left(x_{i}^{-\gamma_{i}-1}\right) \phi\left(\frac{t_{1}}{x_{1}}, \ldots, \frac{t_{r}}{x_{r}}\right)\right\} d x_{1} \ldots d x_{r}
\end{aligned}
$$


then

$$
\begin{aligned}
& \tau\left(s_{1}, \ldots, s_{r}\right)=\frac{1}{\rho_{1} \ldots \rho_{r}} \int_{0}^{\infty} \ldots \int_{0}^{\infty} f\left(x^{\sigma}\right) \\
& \theta\left(s_{1}, \ldots, s_{r}, t_{1}, \ldots, t_{r}, \rho_{1}, \ldots, \rho_{r}\right) d x_{1} \ldots d x_{r}
\end{aligned}
$$

Where

$$
\begin{aligned}
& \theta\left(s_{1}, \ldots, s_{r}, t_{1}, \ldots, t_{r}, \rho_{1}, \ldots, \rho_{r}\right)= \\
& Y\left\{\prod_{i=1}^{r}\left(x_{i}^{\frac{1}{\rho_{i}}-1}\right) g\left(x^{\frac{1}{\rho}}\right) k\left(s x^{\frac{1}{\rho}}\right) ; t_{1}, \ldots, t_{r}\right\}= \\
& \prod_{i=1}^{r}\left(t_{i}^{-\gamma_{i}-1}\right) \int_{0}^{t_{1}} \ldots \int_{0}^{t_{r}} \prod_{i=1}^{r}\left(x_{i}^{\frac{1}{\rho_{i}}+\gamma_{i}-1}\right) g\left(x^{\frac{1}{\rho}}\right) k\left(s x^{\frac{1}{\rho}}\right) \phi\left(\frac{x_{1}}{t_{1}}, \ldots, \frac{x_{r}}{t_{r}}\right) d x_{1} \ldots d x_{r}
\end{aligned}
$$

Where $\rho_{i}$ and $\sigma_{i}(i=1,2, \ldots, r)$ are non zero real numbers of the same sign and all the integrals involved in equations (3.1) to (3.4) are assumed to be absolutely convergent.

Proof: The proof of the above theorem can be easily developed on the lines similar to those of theorem 1 .

Again, if in theorem 2, we replace $\rho_{i}$ by $h_{i}(i=1,2, \ldots, r)$ and take

$$
\begin{aligned}
& g(x)=\prod_{i=1}^{r}\left\{x_{i}^{h_{i}\left(c_{i}+1\right)-1}\right\} \\
& \phi(x)=\prod_{i=1}^{r}\left\{\frac{1}{\Gamma\left(1-\alpha_{i}\right)}{ }_{2} F_{1}\left(\alpha_{i}, \beta_{i}+m ; \beta_{i} ; x_{i}\right)\right\}
\end{aligned}
$$

And $T$ to be multidimensional $I$-function transform defined by (1.8), then proceeding on the lines similar to those of corollary 1 , we obtain the following interesting corollary:

Corollary 2. If $h_{i}>0, \operatorname{Re}\left(1-\alpha_{i}\right)>m, m \in W$, (the set of whole number) $\sigma_{i}(i=1,2, \ldots, r)$ are non-zero real numbers of the same sign.

$\beta_{i} \neq 0,-1,-2, \ldots ; \operatorname{Re}\left(c_{i}\right) \geq 0$

$$
f(x)={ }_{o\left(x_{i}^{B_{i}} e^{-C_{i} x_{i}}\right) \text { forl argevaluesof } x_{i}}^{o\left(x_{i}^{A_{i}}\right) \text { for small valuesof } x_{i}} ; i=1,2, \ldots, r
$$

The multivariable $I$-function satisfy the conditions corresponding appropriately to those given by Srivastava and Panda ([18],p.130, eqs. (1.6) and (1.10)), then

$$
\begin{aligned}
& I\left\{\prod_{i=1}^{r} x_{i}^{h_{i}\left(c_{i}+1\right)-1} \psi\left(x^{h}\right){ }_{p_{2}, q_{2} \ldots p_{r}, q_{r}:\left(p^{\prime}, q\right) \cdots\left(p^{(r)}, q^{(r)}\right),}^{0, n_{2} \cdots n_{n}:\left(m^{\prime}, n\right) \cdots\left(m^{(r)}, n^{(r)}\right),}\right. \\
& \left(a_{2 j}, \alpha_{2 j}^{\prime}, \alpha_{2 j}^{\prime \prime}\right)_{1, p_{2}} \cdots\left(\alpha_{r j}, \alpha_{i j}^{\prime}, \ldots, \alpha_{r j}^{(r)}\right)_{1, p_{r}}:\left(a_{j}^{\prime}, \alpha_{j}^{\prime}\right)_{1, p^{\prime}-1 \ldots} \cdots\left(a_{j}^{(r)}, \alpha_{j}^{(r)}\right)_{1, p^{(r)}-1} \\
& \left(b_{2 j}, \beta_{2 j}^{\prime}, \beta_{2 j}^{\prime}\right)_{1, q_{2}} \cdots\left(b_{r j}, \beta_{r j}^{\prime}, \ldots, \beta_{r j}^{(r)}\right)_{1, q_{r}}:\left(b_{j}^{\prime}, \beta_{j}^{\prime}\right)_{1, q^{\prime}-1 \ldots} \ldots\left(b_{j}^{(r)}, \beta_{j}^{(r)}\right)_{1, q^{(r)}-1} \\
& \left.\begin{array}{l}
\left(-\gamma_{1}-c_{1}, \frac{1}{h_{1}}\right), \ldots,\left(-\gamma_{r}-c_{r}, \frac{1}{h_{r}}\right) \\
\left(\beta_{1}-\gamma_{1}-c_{r}-1, \frac{1}{h_{1}}\right), \ldots,\left(\beta_{r}-\gamma_{r}-c_{r}-1, \frac{1}{h_{r}}\right) ; s_{1}, \ldots, s_{r}
\end{array}\right\}= \\
& \frac{1}{\prod_{i=1}^{r}\left(\beta_{i}\right)_{m}} I\left\{\prod_{i=1}^{r} x_{i}^{h_{i}\left(c_{i}+1\right)-1} f\left(x^{h \sigma}\right) ; \begin{array}{l}
0, n_{2} \cdots 0, n_{r}:\left(m^{\prime}, n\right) \cdots\left(m^{(r)}, n^{(r)}\right), \\
p_{2}, q_{2} \cdots p_{r}, q_{r}:\left(p^{\prime}, q\right) \cdots\left(p^{(r)}, q^{(r)}\right),
\end{array}\right. \\
& \left(a_{2 j}, \alpha_{2 j}^{\prime}, \alpha_{2 j}^{\prime \prime}\right)_{1, p_{2}} \cdots\left(\alpha_{r j}, \alpha_{r j}^{\prime}, \ldots, \alpha_{l j}^{(r)}\right)_{1, p_{r}}:\left(a_{j}^{\prime}, \alpha_{j}^{\prime}\right)_{1, p^{\prime}-1 \cdots} \cdots\left(a_{j}^{(r)}, \alpha_{j}^{(r)}\right)_{1, p^{(r)}-1} \\
& \left(b_{2 j}, \beta_{2 j}^{\prime}, \beta_{2 j}^{\prime}\right)_{1, q_{2}} \cdots\left(b_{r j}, \beta_{r j}^{\prime}, \ldots, \beta_{r j}^{(r)}\right)_{1, q_{r}}:\left(b_{j}^{\prime}, \beta_{j}^{\prime}\right)_{1, q^{\prime}-1 \ldots} \ldots\left(b_{j}^{(r)}, \beta_{j}^{(r)}\right)_{1, q^{(r)}-1}
\end{aligned}
$$

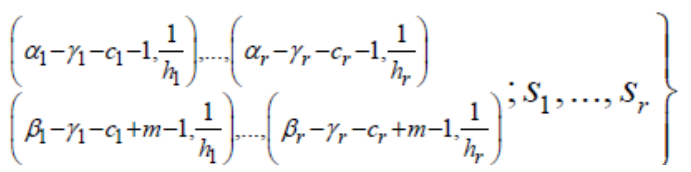


Where

$$
\begin{aligned}
& \psi\left(t_{1}, \ldots, t_{r}\right)=N\left\{f(x) ; t_{1}, \ldots, t_{r}\right\}=\prod_{i=1}^{r}\left\{\frac{t_{i}^{\gamma_{i}}}{\Gamma\left(1-\alpha_{i}\right)}\right\} \\
& \int_{t_{1}}^{\infty} \ldots \int_{t_{r}}^{\infty} \prod_{i=1}^{r}\left\{x_{i}^{-\gamma_{i}-1}{ }_{2} F_{1}\left(\alpha_{i}, \beta_{i}+m ; \beta_{i} ; \frac{t_{i}}{x_{i}}\right)\right\} f\left(x^{\sigma}\right) d x_{1} \ldots d x_{r}
\end{aligned}
$$

Provided that

$$
\begin{aligned}
& \operatorname{Re}\left(c_{i}+\gamma_{i}+\frac{1}{h_{i}} \frac{d_{j}^{(i)}}{D_{i}^{(i)}}\right)>0 ; 1 \leq j \leq m^{(i)} \\
& \operatorname{Re}\left(\sigma_{i} B_{i}+c_{i}+1+\frac{1}{h_{i}} \frac{b_{j}^{(i)}-1}{B_{j}^{(i)}}\right)<0 ; 1 \leq j \leq n^{(i)} ; i=1,2, \ldots, r \\
& \operatorname{Re}\left(\sigma_{i} A_{i}+c_{i}+1+\frac{1}{h_{i}} \frac{d_{k}^{(i)}}{D_{k}^{(i)}}\right)>0 ; 1 \leq k \leq m^{(i)} \\
& \operatorname{Re}\left(\sigma_{i} A_{i}+\beta_{i}-\gamma_{i}+m\right)>0 ; i=1,2, \ldots, r
\end{aligned}
$$

The one and two-dimensional analogous of Theorems 1 and 2 can easily be deduced but since the theorems contain a reasonably detailed analysis of the multidimensional case we prefer to omit their details. The corollaries 1 and 2 given earlier are also new. They give rise to interesting theorems involving multidimensional analogues of fractional integral operators defined by Kober [7], Riemann-Liouville [4] and Weyl [4] and simpler multidimensional integral transforms, on suitably specializing the fractional integral operators and multidimensional $I$-function transform involved therein. Again, the one and two-dimensional analogues of corollaries 1 and 2, yield theorems essentially similar to those given earlier by Gupta, Goyal and Handa ([5],p.165-170), Handa ([6], p. 200-204), Kalla ([8],p. 1008-1010,[9] p. 54-56) and Mathur ([11] p.108-121).

\section{References}

[1]. Erdelyi, A.; On fractional integration and its application to the theory of Hankel transforms, Quart. J. Math. Oxford Ser. (2)2,(1920),293-303.

[2]. Erdelyi, A.; On some fractional transformations, Univ. Politec. Torino. Rend. Sem. Mat. 10, (1950), 217-234.

[3]. Erdelyi, A. et.al.; Higher Transcendental Functions, vol. I, McGraw-Hill, New York (1953).

[4]. Erdelyi, A. et.al.; Tables of Integral Transforms, vol. II, McGraw-Hill, New York (1954).

[5]. Gupta, K.C., Goyal, S.P. and Handa, S.; Fractional integral operators involving two variables, Univ. Nac. Tucuman Rev. Ser. A 26 ,(1976), 159-171.

[6]. Handa, S.; A study of generalized functions of one and two variables, Indian J. of Math., (1976), 11-16.

[7]. Kober, H.; On fractional integrals and derivatives, Quart. J.Math. Oxford Ser. 2(II), (1940), 193-211.

[8]. Kalla, S.L.; Some theorems on fractional integration, Proc. Nat. Acad. Sci. India Sect. A 36, (1966), 1007-1012.

[9]. Kalla, S.L.; Some theorems on fractional integration II, Proc. Nat. Acad. Sci. India Sect. 39, (1969), 44-56.

[10]. Kalla, S.L.; Fractional integration operators involving generalized hypergeometric functions, Univ. Nac. Tucuman. Rev. Ser. A 20, (1970), 93-100.

[11]. Mathur, S.L.; Astudy of boundary value problems and special functions, Proc. Acad. Sci India Sect.A 43,(1974),56-61.

[12]. Pasad, Y.N.; On a multivariable I-function, Vijnana Parishad Anuusandhan Patrika, vol.29(4),(1986), 231-235.

[13]. Saxena, R.K.; On fractional integral operators, Math. Zeitscher. 95, (1967),288-291. 
[14]. Saxena, R.K. and Modi, G.C.; Multidimensional fractional integration operators associated with hypergeometric kernels, Nat. Acad. Sci. Lett. 13, (1980), 153-158.

[15]. Sneddon, I.N.; Mixed Boundary Value Problems in Potential Theory, North-Holland Publishing Co. , Amsterdam (1966).

[16]. Srivastava, H.M. and Buschmann R.G.; Composition of fractional integral operators involving Fox's H-function, Acta Mexicana Ci Tecn 7, (1973), 21-28.

[17]. Srivastava, H.M. and Panda, R.; Some bilateral generating functions for a class of generalized polynomials, J. Reine Angew Math. 283/284, (1976), 265-274.

[18]. Srivastava, H.M. and Panda, R.; Expansion theorems for the H-function of several complex variables, J.Reine Angew Math 288, (1976), 129-145.

[19]. Srivastava, H.M. and Panda, R.; Certain multidimensional integral transformations I and II, Nederl. Akad Wetensch Proc. Ser. A 81, (1978), 118-141.

[20]. Srivastava, H.M. , Gupta, K.C. and Goyal, S.P.; The H-function of One and Two Variables With Applications, South Asian Publishers Pvt. Ltd., New Delhi and Madras (1982). 